\title{
A Caenorhabditis elegans genetic-interaction map wiggles into view Kristin C Gunsalus
}

Address: Center for Genomics and Systems Biology and Department of Biology, New York University, 1009 Silver Center, 100 Washington Square East, New York, NY 10003, USA. Email: kcg1@nyu.edu

Published: 7 March 2008

Journal of Biology 2008, 7:8 (doi: 10.1 186/jbiol70)

The electronic version of this article is the complete one and can be found online at http://jbiol.com/content/7/3/8

(c) 2008 BioMed Central Ltd

\begin{abstract}
Systematic mapping of genetic-interaction networks will provide an essential foundation for understanding complex genetic disorders, mechanisms of genetic buffering and principles of robustness and evolvability. A recent study of signaling pathways in Caenorhabditis elegans lays the next row of bricks in this foundation.
\end{abstract}

One of the enduring challenges in biology is to learn how the amazing complexity and diversity of life forms arise from a limited repertoire of heritable factors. To understand the emergent properties of biological systems, it is necessary to first map the functional organization of the complex biological networks that underlie them. Many levels of function will need to be analyzed systematically to arrive at this goal. Mapping molecular interactions such as proteinprotein, protein-DNA, and RNA-RNA interactions will help define structural and regulatory relationships. However, understanding organizational principles that determine how different parts of these networks are coordinated will require uncovering functional dependencies that may not be reflected in direct physical interactions, for example between actin- and tubulin-dependent cellular processes [1]. Large-scale mapping of genetic interactions in model organisms offers a powerful approach to tackle this challenge. A recent genetic-interaction study published in Journal of Biology by Byrne et al. [2], focusing on signaling pathways of the nematode worm Caenorhabditis elegans, pushes the envelope of genetic-interaction mapping in a multicellular organism by developing a novel approach to defining networks of genetic interactions based on interaction strength, and integrating these networks with other dimensions of genome-scale data in order to reveal global patterns of functional relationships.

\section{Unraveling the functional organization of biological networks}

Why is it important to gain a global view of genetic interactions? One simple reason is to help assign functions to the many nonessential genes whose in vivo requirements remain obscure. Genetic and reverse genetic studies in Saccharomyces cerevisiae [3], C. elegans [4-7], and Drosophila melanogaster [8] indicate that the majority of genes (around $75-85 \%$ ) in both single-celled eukaryotes and metazoans appear to be dispensable for survival; moreover, only about half of protein-coding genes in yeast [3,9] and about $25 \%$ in the worm [10] give rise on their own to any discernable phenotype in vivo. However, genetic modifier screens for enhancement or suppression of specific phenotypes have been used with great success in model organisms to identify genes with related functions and to order genes within pathways involved in numerous biological processes (for a review see [11]). Many genetic elements identified in this way give rise to detectable phenotypes only when their function is compromised in combination with other genetic loci. In medicine, there is an increasing recognition that the etiology of many diseases involves multiple genetic factors that confound simple genotype-phenotype relationships [12].

Characterizing patterns of genetic interactions can also help us understand how organisms resist or adapt to environ- 
mental or genetic variation. Biological networks are increasingly seen as modular systems [13], in which coordinated assemblies of components with specialized functions mediate distinct processes that are, to some extent, insulated from other parts of the network. Thus perturbing the activity of a single component is often not catastrophic; instead, systems find ways to compensate. This impressive resilience is thought to reflect fundamental architectural properties of molecular networks that underlie both the robustness and the adaptability of biological systems. Robustness refers to the ability of organisms to maintain phenotypic stability through homeostatic mechanisms that allow them to tolerate fluctuations in environmental conditions or genetic variation [14]. Phenotypic buffering allows the accumulation of mutations in a particular genetic background; when buffering mechanisms break down, this hidden genetic variation may become expressed. This is famously illustrated by the example of HSP90 [15] - which when impaired can release striking morphological diversity in almost any adult structure in the fly - but may be a more general property of genetic networks [16]. The release of phenotypic variation has important implications for evolutionary change $[17,18]$. Thus, buffering can both promote homeostasis and foster phenotypic plasticity under the right conditions. Identifying functional connections between particular molecules and modules on a global scale will help us both to learn about explicit mechanisms and to develop a theoretical framework for how organisms adjust to variability in external conditions and internal network states.

\section{Insights from genetic networks in yeast}

The most comprehensive analyses of genetic interactions so far have been performed in S. cerevisiae. High-throughput approaches have been developed in yeast to create qualitative and quantitative maps of genetic interactions, including synthetic sick or lethal (SSL) interactions for essential and nonessential sets of genes, synthetic dosage suppression or lethality, and complex haploinsufficient interactions [19]. These techniques are enabled by the generation of strain libraries with mutations in every gene, allowing large-scale screening of deletions, conditional or hypomorphic alleles and inducible overexpression constructs [19]. These approaches have also been extended to map the sensitivity of yeast to various chemicals, revealing interactions between specific genes and environmental perturbations (see, for example, [20-22]).

The growing body of genetic-interaction studies has greatly extended our understanding of the functional organization of biological processes in yeast, in terms of both specific functional relationships and global properties [19]. For example, although the SSL and protein-protein interaction (PPI) maps overlap more than expected by chance (approximately $13 \%$ of within-complex PPIs are SSLs, compared with $0.5 \%$ expected by chance), the number of overlapping interactions is very small overall (around 1-4\% of SSL pairs are also PPIs), pointing to essential differences in the type of information that these networks provide about functional organization within cells [1]. PPIs correspond mainly to physical complexes and pathways, whereas patterns of SSL interactions predominantly reveal between-pathway relationships that expose functional links between related cellular processes; thus genes in the same pathway or complex tend to share many of the same genetic-interaction partners [1].

This body of data has also stimulated significant interest in exploring the types of interactions that can be observed genetically [23] and in defining mathematical models that should be applied to interpret the results of geneticinteraction studies [24]. For example, using a 'min' definition, in which any phenotype worse than either of the single mutants is called a genetic interaction, will yield a different (and much larger) set of interactions than using a 'product' rule, in which the phenotype of a double mutant must be worse than the product of either single mutant alone [24]. Considering synergistic genetic interactions in yeast, alternative definitions differ with respect to identifying functional relationships and can lead to different conclusions regarding the underlying biology [24]. This issue also has significant implications for the interpretation of genetic interactions in other organisms.

\section{Mapping genetic networks in $C$. elegans}

Similar approaches now need to be extended to study complex interactions in multicellular organisms. As described in Byrne et al. [2], a collaborative study between the groups of Peter Roy and Josh Stuart takes a significant new step in this direction. Although the analysis of genetic interactions for individual genes of interest has long been a mainstay of genetics in metazoan model organisms such as the worm and the fly, large-scale systematic efforts have lagged far behind those in yeast, mainly because of technical limitations: comprehensive libraries of deletion strains do not yet exist, and selecting and analyzing progeny from the 200 million or so possible mutant crosses using forward genetic methods is a logistical nightmare. With few reported exceptions [25], a purely reverse genetic approach using combinatorial RNA interference (RNAi) to target two genes simultaneously in the same animal has not met with great success in most worm labs. However, a hybrid strategy, in which individual genetic alleles are screened against a library of genes depleted one at a time by RNAi, has proved an effective alternative in studies of increasing 
scale [26-29]. Using a hybrid genetic-RNAi approach, Byrne et al. [2] report a network of 1,246 genetic interactions between genetic alleles of 11 'query' genes (primarily involved in conserved signaling pathways specific to metazoans) and genes from a library of 858 'target' genes depleted individually by RNAi. The target gene set was split between 372 genes likely to be involved in signal transduction (based on functional annotations) and 486 genes on linkage group III (which may contain new, previously unidentified signaling targets).

Although the total number of interactions tested was not significantly larger than several recent studies [27,29-31], the work by Byrne et al. [2] stands out in its attempt to provide a more quantitative assessment of the strength of genetic interactions and in its novel use of a global dataanalysis approach designed to identify interacting pairs in an unbiased fashion. The experimental design involved estimating numbers of progeny on solid agar over several days using a graded scoring scheme in blind triplicate assays. From these data the authors constructed a large compendium matrix of 56,347 scores and inferred 51 unique sets of genetic interactions by varying six parameters (for example, deviation between experimental and control samples, number of days with an observed deviation and reproducibility). They then chose two network variants that corresponded best to shared Gene Ontology (GO) terms [32]: a 'high confidence' variant containing 656 unique interactions among 253 genes, and a larger variant with slightly higher recall containing 1,246 interactions among 461 genes.

What lessons did Byrne et al. [2] learn from this study? To evaluate their results, the authors analyzed their geneticinteraction networks in a variety of ways, both independently and in combination with other datasets. First, they identified many potential new functional links and confirmed a number of previously noted links within and between specific signaling pathways (for example, transforming growth factor $\beta \leftrightarrow \mathrm{Wnt} / \beta$-catenin; fibroblast growth factor $\leftrightarrow$ epidermal growth factor). These links provide many hypotheses for follow-up studies to determine their potential significance in development. Second, based on comparisons with a variety of other datasets, Byrne et al. concluded that their approach resulted in much higher detection sensitivity than most previous screens, which they attributed to their ability to detect both strong and weak interactions and their novel method of identifying interacting pairs. Third, by overlaying their geneticinteraction network with protein-protein interactions, coexpression and co-phenotype data, the authors found that there is little overlap between datasets, suggesting that the genetic interactions they identified are revealing novel

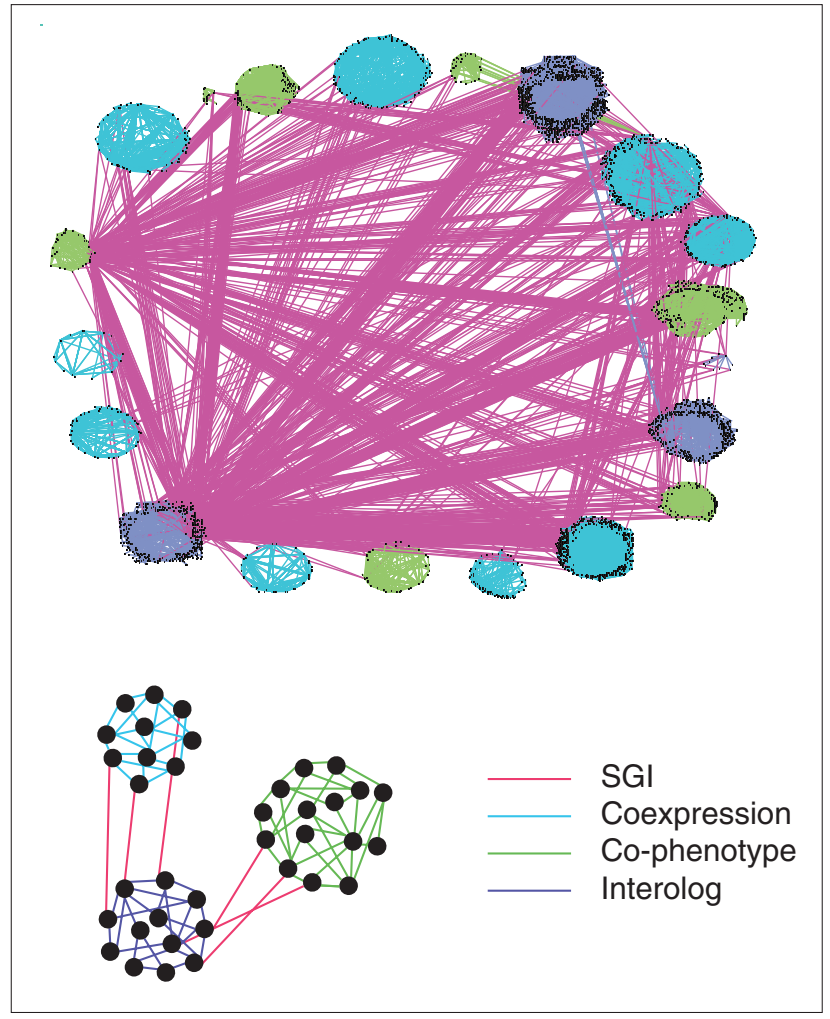

Figure I

Adapted from Byrne et al. [2], a superimposed network composed of different types of functional linkages contains subnetworks of genes that are highly interconnected based on one type of data: coexpression (blue), co-phenotype (green), or eukaryotic protein-protein interactions ('interolog'; purple). Byrne et al. found that these subnetworks were bridged by genetic interactions (pink) more often than expected by chance. Many such subnetworks were enriched for genes with shared functional annotations, supporting the idea that enhancing genetic interactions (identified by reduced function of a pair of genes) tend to bridge distinct functional modules.

functional relationships. Even though the PPI and phenotype data are still relatively sparse with respect to the entire genome, and the level of specificity provided by the phenotype and expression links is limited, this result is consistent with studies in yeast.

Within the superimposed network, the authors identified highly connected subnetworks, which in at least one example revealed a significant enrichment for similar RNAi phenotypes and previously undocumented genetic interactions upon retesting. Many of these subnetworks were enriched for shared functional annotations, and a significant number were bridged by genetic interactions (Figure 1), supporting the idea that genetic interactions connect different functional modules. This observation is curious in light of the fact that the final genetic-interaction 
network was selected to maximize shared GO terms, and possibly suggests that this standard may not be the optimal measure to evaluate the fine structure of functional relationships within a cell or organism. Alternatively, refining the functional neighborhoods used for this analysis ('broad subnetworks' based on a single mode of interaction, such as coexpression, and containing dozens or hundreds of genes) may provide a higher level of resolution that would bring these relationships into better focus. Finally, when the authors compared the connectivity of yeast [1,33] and worm [2,27] geneticinteraction networks, they found no significant evidence for conservation of synthetic genetic interactions between species. Thus, as in yeast [1], genetic interactions identified in the worm appear to reveal higher-level intermodule functional relationships (see Figure 1); however, the specific patterns of connectivity between modules may not be evolutionarily conserved.

\section{Looking to the future}

These are very early days for systematic genetic interaction studies in metazoans, and many questions - both theoretical and technical - remain unresolved. A notably unglamorous but important set of technical considerations is that differences in methodology between different studies in the same organism will heavily influence both the composition of reported datasets and conclusions drawn from them. Chief among these considerations, as illustrated by the 51 network variants identified by Byrne et al. [2] and comparisons with results from a similar study by Lehner et al. [27], is that differences in experimental design, scoring methods and models used to define genetic interactions [24] will necessarily result in different sets of reported interactions. It is not yet clear how to evaluate these differences. Notably, both Byrne et al. and Lehner et al. achieved high technical reproducibility ( $83 \%$ and more than $90 \%$, respectively); in contrast, when genetic alleles and RNAi for query-target pairs were reversed, only $40 \%$ (6/15) of reciprocal tests by Byrne et al. interacted. This indicates that these screens may be far from saturation, as RNAi does not always phenocopy genetic alleles and can carry considerable false-negative rates [34]. Unlike Lehner et al. [27], who placed a lower estimate of $32 \%$ on their detection rate for previously reported genetic interactions (some of which, for example suppressors, would not be expected to be detected as synthetic lethals), Byrne et al. [2] did not compare their results with a 'gold standard' of genetic interactions from the literature. Instead, they evaluated functional cohesion by precision and recall of shared GO terms, achieving somewhat lower precision but much higher recall (as well as a higher total number of interactions) among pairs tested in both studies. This and other comparisons suggest that the detection methods used by Lehner et al. [27] were more stringent, resulting in a bias toward stronger genetic interactions, and that Byrne et al. [2] cast a much wider net for recovery of genetic interactions.

A further improvement over the semi-quantitative scoring approach used by Byrne et al. [2], which was based on binned ranges of estimated survival rates, would be to precisely measure lethality in these assays. Currently, one of the biggest technical limitations for large-scale RNAi-based screens in C. elegans is the lack of efficient high-throughput methods to quantitate lethality, growth rates, and other morphological phenotypes, which limits the extent to which issues surrounding the quantitative definition of genetic interactions $[23,24]$ can be explored. Over time, as technical approaches evolve and further large-scale screens and in-depth studies accumulate, it will be interesting to revisit these comparisons.

A more profound question is, to what extent will patterns of genetic interactions be conserved across species? Answers to this question will inform how we use crossspecies inferences to guide studies in less experimentally tractable systems. A preliminary comparison between worm and yeast [2] suggested that, in contrast to PPIs, there is little conservation of genetic interactions between these two organisms. This conclusion is clouded, however, by caveats on several levels. For example, it is not clear if this comparison considered whether all of the positive genetic interaction pairs in C. elegans were actually tested in yeast. Since the set of gene pairs that has been tested differs substantially between yeast and worm, tests of conservation should be made only for subsets of gene pairs that have been systematically tested in both organisms. More obvious is the dichotomy between unicellular and multicellular organisms: yeast are directly exposed to the environment, and must modulate their internal states accordingly, whereas metazoans comprise many different cell types with distinct internal states and external contacts. Measuring survival and growth rates thus provides a relatively direct readout of cell status in yeast, whereas the types of phenotypic assay performed in metazoans will heavily influence our ability to detect different patterns of genetic interactions. The interpretation of negative results in whole-animal assays is further complicated by the possibility - given a particular experimental setup or phenotypic assay - that two potentially interacting components may not become limiting in the same cell types, or that interactions in a subset of cells will not give rise to obvious organismal phenotypes. Studies of mammalian and Drosophila cells in culture have begun to report genome-wide genetic requirements for specific cellular functions $[35,36]$, but these cannot reveal how biological systems as a whole adapt to the loss of specific 
genetic determinants. Thus, the answer to whether geneticinteraction studies in model systems will provide practical insights into human biology and disease mechanisms awaits further studies. Good reason for optimism stems from the deep conservation of many developmental signaling pathways and the fact that many human disease processes can be effectively studied in these models (the fly and worm, for example, even provide model systems to study mechanisms underlying Alzheimer's disease [37]).

What's next? Extending systematic genetic-interaction maps to other metazoan systems, including alleviating (suppressing) as well as synthetic (enhancing) interactions, using more specific high-throughput assays (for example, those that allow tissue-specific readouts [38]), and developing quantitative assays, will greatly expand our understanding of molecular network organization in complex multicellular organisms. These approaches could also be combined with chemical genetic profiling, as pioneered in yeast $[21,22]$, to develop therapeutic strategies based on multiple molecular targets within the cell. Experimental approaches for mapping genetic interactions will both inform and be guided by efforts to generate predictive models for both gene function and functional associations between genes (for example $[39,40]$ ): the continued accumulation of large unbiased training sets will help develop better predictive methods, which in turn will help fill out neighborhoods of interactions and reduce the combinatorial search space for studies directed at specific pathways. Finally, it will be interesting to compare the spectrum of phenotypes and genetic interactions identified in systematic studies of genetic alleles and RNAi with those arising from variation in natural populations (for example, see [41]). Building on knowledge gained from decades of studying specific genes and pathways, global analysis of genetic-interaction networks promises to reveal new insights that will broadly influence our thinking about both applications to medicine and the relationship between network architecture and biological function.

\section{Acknowledgements}

I wish to thank F. Roth, M. Siegal, F. Piano and A. Fernandez for constructive comments on the manuscript and NIH (HD046236 and HG004276), US Department of the Army (W23RYX-3275-N605), and NYSTAR (C040066) for research support.

\section{References}

I. Tong AH, Lesage G, Bader GD, Ding H, Xu H, Xin X, Young J, Berriz GF, Brost RL, Chang M, Chen Y, Cheng X, Chua G, Friesen H, Goldberg DS, Haynes J, Humphries C, He G, Hussein S, Ke L, Krogan N, Li Z, Levinson JN, Lu H, Ménard P, Munyana C, Parsons AB, Ryan $\mathrm{O}$, Tonikian R, Roberts $\mathrm{T}$, et al:: Global mapping of the yeast genetic interaction network. Science 2004, 303:808-813.

2. Byrne AB, Weirauch MT, Wong V, Koeva M, Dixon SJ, Stuart JM, Roy PJ: A global analysis of genetic interactions in Caenorhabditis elegans. J Biol 2007, 6:8.
3. Giaever G, Chu AM, Ni L, Connelly C, Riles L, Véronneau S, Dow S, Lucau-Danila A, Anderson K, André B, Arkin AP, Astromoff A, El-Bakkoury M, Bangham R, Benito R, Brachat S, Campanaro S, Curtiss M, Davis K, Deutschbauer A, Entian KD, Flaherty P, Foury F, Garfinkel DJ, Gerstein M, Gotte D, Güldener U, Hegemann JH, Hempel S, Herman Z, et al.: Functional profiling of the Saccharomyces cerevisiae genome. Nature 2002, 418:387-391.

4. Kamath RS, Fraser AG, Dong Y, Poulin G, Durbin R, Gotta M, Kanapin A, Le Bot N, Moreno S, Sohrmann M, Welchman DP, Zipperlen P, Ahringer J: Systematic functional analysis of the Caenorhabditis elegans genome using RNAi. Nature 2003, 42I: 23I-237.

5. Rual JF, Ceron J, Koreth J, Hao T, Nicot AS, Hirozane-Kishikawa T, Vandenhaute J, Orkin SH, Hill DE, van den Heuvel S, Vidal M: Toward improving Caenorhabditis elegans phenome mapping with an ORFeome-based RNAi library. Genome Res 2004, 14:21622168.

6. Simmer F, Moorman C, van der Linden AM, Kuijk E, van den Berghe PV, Kamath RS, Fraser AG, Ahringer J, Plasterk RH: Genome-wide RNAi of $C$. elegans using the hypersensitive rrf-3 strain reveals novel gene functions. PLOS Biol 2003, I:EI2.

7. Sönnichsen B, Koski LB, Walsh A, Marschall P, Neumann B, Brehm M, Alleaume AM, Artelt J, Bettencourt P, Cassin E, Hewitson M, Holz C, Khan M, Lazik S, Martin C, Nitzsche B, Ruer M, Stamford J, Winzi M, Heinkel R, Röder M, Finell J, Häntsch H, Jones SJ, Jones M, Piano F, Gunsalus KC, Oegema K, Gönczy P, Coulson A, et al:: Full-genome RNAi profiling of early embryogenesis in Caenorhabditis elegans. Nature 2005, 434:462-469.

8. Ashburner M, Misra S, Roote J, Lewis SE, Blazej R, Davis T, Doyle C, Galle R, George R, Harris N, Hartzell G, Harvey D, Hong L, Houston K, Hoskins R, Johnson G, Martin C, Moshrefi A, Palazzolo M, Reese MG, Spradling A, Tsang G, Wan K, Whitelaw K, Kimmel B, Celniker S, Rubin GM: An exploration of the sequence of a 2.9-Mb region of the genome of Drosophila melanogaster: the Adh region. Genetics 1999, I53:179-219.

9. Ohya Y, Sese J, Yukawa M, Sano F, Nakatani Y, Saito TL, Saka A, Fukuda T, Ishihara S, Oka S, Suzuki G, Watanabe M, Hirata A, Ohtani M, Sawai H, Fraysse N, Latgé JP, François JM, Aebi M, Tanaka S, Muramatsu S, Araki H, Sonoike K, Nogami S, Morishita S: High-dimensional and large-scale phenotyping of yeast mutants. Proc Natl Acad Sci USA 2005, 102:19015-19020.

10. Rogers A, Antoshechkin I, Bieri T, Blasiar D, Bastiani C, Canaran P, Chan J, Chen WJ, Davis P, Fernandes J, Fiedler TJ, Han M, Harris TW, Kishore R, Lee R, McKay S, Müller HM, Nakamura C, Ozersky P, Petcherski A, Schindelman G, Schwarz EM, Spooner W, Tuli MA, Van Auken K, Wang D, Wang X, Williams G, Yook K, Durbin R, et al.: WormBase 2007. Nucleic Acids Res 2008, 36(Database issue):D6I2-D6I7.

11. Jorgensen EM, Mango SE: The art and design of genetic screens: Caenorhabditis elegans. Nat Rev Genet 2002, 3:356-369.

12. Badano JL, Katsanis N: Beyond Mendel: an evolving view of human genetic disease transmission. Nat Rev Genet 2002, 3:779-789.

13. Hartwell LH, Hopfield J], Leibler S, Murray AW: From molecular to modular cell biology. Nature 1999, 402:C47-C52.

14. Hartman JL 4th, Garvik B, Hartwell L: Principles for the buffering of genetic variation. Science 200I, 29I: I00 I- 1004.

15. Rutherford SL, Lindquist S: Hsp90 as a capacitor for morphological evolution. Nature 1998, 396:336-342.

16. Bergman A, Siegal ML: Evolutionary capacitance as a general feature of complex gene networks. Nature 2003, 424:549-552.

17. Rutherford SL: From genotype to phenotype: buffering mechanisms and the storage of genetic information. BioEssays 2000, 22:1095-1105.

18. Wagner A: Robustness and evolvability: a paradox resolved. Proc Biol Sci 2008, 275:9I-I00.

19. Boone C, Bussey H, Andrews BJ: Exploring genetic interactions and networks with yeast. Nat Rev Genet 2007, 8:437-449.

20. Hartman JL 4th, Tippery NP: Systematic quantification of gene interactions by phenotypic array analysis. Genome Biol 2004, 5:R49.

21. Lum PY, Armour CD, Stepaniants SB, Cavet G, Wolf MK, Butler JS, Hinshaw JC, Garnier P, Prestwich GD, Leonardson A, Garrett-Engele P, 
Rush CM, Bard M, Schimmack G, Phillips JW, Roberts C], Shoemaker DD: Discovering modes of action for therapeutic compounds using a genome-wide screen of yeast heterozygotes. Cell 2004, II6:121-137.

22. Parsons AB, Lopez A, Givoni IE, Williams DE, Gray CA, Porter J, Chua G, Sopko R, Brost RL, Ho CH, Wang J, Ketela T, Brenner C, Brill JA, Fernandez GE, Lorenz TC, Payne GS, Ishihara S, Ohya Y, Andrews B, Hughes TR, Frey BJ, Graham TR, Andersen RJ, Boone C: Exploring the mode-of-action of bioactive compounds by chemicalgenetic profiling in yeast. Cell 2006, I26:6 I I-625.

23. Drees BL, Thorsson V, Carter GW, Rives AW, Raymond MZ, Avila-Campillo I, Shannon P, Galitski T: Derivation of genetic interaction networks from quantitative phenotype data. Genome Biol 2005, 6:R38.

24. Mani R, St Onge RP, Hartman JL 4th, Giaever G, Roth FP: Defining genetic interaction. Proc Natl Acad Sci USA 2008, doi:I0.1073/pnas.07|2255I05

25. Tischler J, Lehner B, Chen N, Fraser AG: Combinatorial RNA interference in Caenorhabditis elegans reveals that redundancy between gene duplicates can be maintained for more than $\mathbf{8 0}$ million years of evolution. Genome Biol 2006, 7:R69.

26. Baugh LR, Wen JC, Hill AA, Slonim DK, Brown EL, Hunter CP: Synthetic lethal analysis of Caenorhabditis elegans posterior embryonic patterning genes identifies conserved genetic interactions. Genome Biol 2005, 6:R45.

27. Lehner B, Crombie C, Tischler J, Fortunato A, Fraser AG: Systematic mapping of genetic interactions in Caenorhabditis elegans identifies common modifiers of diverse signaling pathways. Nat Genet 2006, 38:896-903.

28. Suzuki $Y$, Han M: Genetic redundancy masks diverse functions of the tumor suppressor gene PTEN during $C$. elegans development. Genes Dev 2006, 20:423-428.

29. van Haaften G, Vastenhouw NL, Nollen EA, Plasterk RH, Tijsterman M: Gene interactions in the DNA damage-response pathway identified by genome-wide RNA-interference analysis of synthetic lethality. Proc Natl Acad Sci USA 2004, I0I: 12992-12996.

30. Labbé JC, Pacquelet A, Marty T, Gotta M: A genomewide screen for suppressors of par-2 uncovers potential regulators of PAR protein-dependent cell polarity in Caenorhabditis elegans. Genetics 2006, I74:285-295.

31. O'Rourke SM, Dorfman MD, Carter JC, Bowerman B: Dynein modifiers in $C$. elegans: light chains suppress conditional heavy chain mutants. PLoS Genet 2007, 3:e 128.

32. Ashburner M, Ball CA, Blake JA, Botstein D, Butler H, Cherry JM, Davis AP, Dolinski K, Dwight SS, Eppig JT, Harris MA, Hill DP, Issel-Tarver L, Kasarskis A, Lewis S, Matese JC, Richardson JE, Ringwald M, Rubin GM, Sherlock G: Gene ontology: tool for the unification of biology. The Gene Ontology Consortium. Nat Genet 2000, 25:25-29.

33. Breitkreutz BJ, Stark C, Reguly T, Boucher L, Breitkreutz A, Livstone M, Oughtred R, Lackner DH, Bähler J, Wood V, Dolinski K, Tyers M: The BioGRID Interaction Database: 2008 update. Nucleic Acids Res 2008, 36:D637-D640.

34. Fernandez AG, Gunsalus KC, Huang J, Chuang LS, Ying N, Liang HL, Tang C, Schetter AJ, Zegar C, Rual JF, Hill DE, Reinke V, Vidal M, Piano F: New genes with roles in the $C$. elegans embryo revealed using RNAi of ovary-enriched ORFeome clones. Genome Res 2005, 15:250-259.

35. Moffat J, Sabatini DM: Building mammalian signalling pathways with RNAi screens. Nat Rev Mol Cell Biol 2006, 7:177-187.

36. Perrimon N, Mathey-Prevot B: Applications of high-throughput RNA interference screens to problems in cell and developmental biology. Genetics 2007, 175:7-16.

37. Link CD: Invertebrate models of Alzheimer's disease. Genes Brain Behav 2005, 4: 147-156.

38. Ni JQ, Markstein M, Binari R, Pfeiffer B, Liu LP, Villalta C, Booker M, Perkins L, Perrimon N: Vector and parameters for targeted transgenic RNA interference in Drosophila melanogaster. Nat Methods 2008, 5:49-5।.

39. Lee I, Lehner B, Crombie C, Wong W, Fraser AG, Marcotte EM: A single gene network accurately predicts phenotypic effects of gene perturbation in Caenorhabditis elegans. Nat Genet 2008, 40:181- 188.
40. Zhong W, Sternberg PW: Genome-wide prediction of C. elegans genetic interactions. Science 2006, 3 I I: | 48 I- I 484

4I. Seidel HS, Rockman MV, Kruglyak L: Widespread genetic incompatibility in C. elegans maintained by balancing selection. Science 2008, 319:589-594. 